\title{
Fault Tolerant Control for Non-Gaussian Stochastic Distribution Systems
}

\author{
Yi Qu • Zhan-Ming Li • Er-Chao Li
}

Received: 14 January 2012 / Revised: 1 June 2012 / Published online: 19 June 2012

(C) The Author(s) 2012. This article is published with open access at Springerlink.com

\begin{abstract}
A new fault tolerant control (FTC) problem via the output probability density functions (PDFs) for non-Gaussian stochastic distribution control systems (SDC) is investigated. The PDFs can be approximated by the radial basis functions (RBFs) of neural networks. Differently from the conventional FTC problems, the measured information is in the form of probability distributions of the system output rather than the actual output values. The control objective is to use the output PDFs to design control algorithm that can compensate the faults and attenuate the disturbances. As a result, the concerned FTC problem subject to dynamic relation between the input and output PDFs can be transformed into a nonlinear FTC problem subject to dynamic relation between the control input and the weights of the RBFs neural networks. Feasible criteria to compensate the faults and attenuate the disturbances are provided in terms of linear matrix inequality (LMI) techniques. In order to improve FTC performances, $H^{\infty}$ optimization techniques are applied to the FTC design problem to assure that the faults can be compensated and the disturbances can be attenuated. At last, an illustrated example is given to demonstrate the efficiency of the proposed algorithm, and the satisfactory results have been obtained.
\end{abstract}

Keywords Fault tolerant control · Probability density functions · Non-Gaussian stochastic distribution control systems · Radial basis functions neural networks · Linear matrix inequality

Y. Qu (凶) · Z.-M. Li · E.-C. Li

College of Electrical and Information Engineering, Lanzhou University of Technology, No. 287, Langongping Road, Qilihe District, Lanzhou, Gansu, 730050, P.R. China e-mail: quyi0709052@163.com

Z.-M. Li

e-mail: Lizm@lut.cn

E.-C. Li

e-mail: lecstarr@163.com 


\section{Introduction}

In the past three decades, the research on design of controllers for stochastic systems has been regarded as an important aspect in control theory and practice. Fault detection and diagnosis (FDD) and fault tolerant control (FTC) theory have attracted the considerable academic interest and, as a result, a variety of techniques for FDD and FTC have been developed during the last 20 years. For stochastic systems, the two kinds of approaches, including the system identification techniques $[2,5,14,15,17,27,35]$ and the statistic approaches based on the Likelihood methods, Bayesian theory, and Hypothesis test techniques [27], can be used to deal with the related FDD problems. Besides, it is known that filter-based methods have been extensively applied to generate the residual signal for the fault detection and diagnosis $[9,18,26,30]$, and many of their significant approaches have been successfully applied to practical processes $[18,30]$.

It is noted that most of the FDD methodologies for stochastic systems only investigated Gaussian systems [2, 5, 11, 14-17, 27, 35], and one of the common features for these methods is performed by using system input and output measuring values. However, in many practical processes, non-Gaussian variables exist in stochastic systems due to nonlinearity, and these may possess asymmetric and multiple-peak stochastic distributions, where mean and variance are insufficient to precisely characterize their statistical behaviors. With the development of instruments and image processing techniques, the measured information can be the stochastic distribution of system output distribution rather than its instant values. So there is a need to further develop the FDD methods that can be applied to the stochastic systems subject to non-Gaussian distribution. Motivated by these factors, studies on stochastic distribution systems and stochastic distribution control have been investigated in $[1,3,4,6,7,10,19,20,22-25,31-33]$. Differently from conventional FDD problems, the measurement information for the FDD is the output PDFs rather than the mean or variance of the output, and the stochastic variables involved in are not confined to the Gaussian ones.

Up to now, many effective fault detection and diagnosis methods for non-Gaussian stochastic distribution systems have been considered by researchers to cover various types of faulty systems [1, 4, 6-13, 19, 20, 22, 24, 25, 31-34]. However, FDD is only the first step in the reliable control procedures.

Fault tolerant control is in using the control input and the measured information to design the controller so that the system can work normally even when the fault occurs. One of the feasible methods is to design the filter to detect and diagnose the fault, and then to configure the controller to compensate or refuse to accept the estimation of the fault. Compared with the FDD, the virtual problem is to use the measured output PDFs to provide FTC strategies for non-Gaussian stochastic distribution control systems. In most cases, the literature on the FTC methods for the stochastic systems has been provided under the assumption that the random variables or noise are confined to be Gaussian distribution [1, 4, 6-8, 12, 13, 19, 25, 34]. Up to now, it is noted that few available literatures for non-Gaussian stochastic distribution control systems have been presented in the FTC context. As a result, there is a need to develop the FTC methods that can be applied to the non-Gaussian stochastic distribution control 
systems, and the new filtering algorithms are required. This is the main purpose of the paper.

In this work, the radial basic functions (RBFs) models are used to address fault tolerant control (FTC) problems. It can be seen that the concerned problem can be reduced to a nonlinear FTC problem. To improve the performance of the FTC for the non-Gaussian stochastic distribution control systems, the proportional integral derivative (PID) controller and $H^{\infty}$ optimization techniques are introduced in the presence of both the fault and the system disturbance, the control objective being to use the system output information (PDFs) to construct controller that can attenuate the disturbance and compensate the fault. Finally, a linear matrix inequality based on the feasible FTC method is given so that the fault can be compensated or rejected.

This paper is organized as follows. In Sect. 2, the output PDFs expansion and the nonlinear weight dynamic are established to formulate the FTC problem. The FTC filtering is designed to compensate or reject faults in Sect. 3. In Sect. 4, a simple example is given to demonstrate the efficiency of the proposed approach. Finally, concluding remarks are made in Sect. 5.

\section{Problem Formulation}

Consider a continuous-time dynamic stochastic distribution systems where $u(t) \in R^{m}$ is the control input, $y(t) \in[a, b]$ represents the system output, and $F$ is the fault to be compensated or rejected, a typical example of which is an actuator fault. At any time, the probability of output $y(t)$ lying inside $[a, b]$ can be described as follows:

$$
P(a \leq y(t)<b)=\int_{a}^{b} \gamma(y, u(t), F) d y
$$

where $\gamma(y, u(t), F)$ denotes the PDF of the stochastic variable $y(t)$ under the control input signal $u(t)$. The control objective in the FTC context is to use the output PDFs to design controller that can compensate or reject the fault. As shown in [20], the well-known RBF neural network has been used approximately by the following expression:

$$
\sqrt{\gamma(y, u(t), F)}=\sum_{i=1}^{n} v_{i}(u(t), F) b_{i}(y)+\omega_{0}(y, u(t), F)
$$

where $b_{i}(y)(i=1,2, \ldots, n)$ represents the $i$ th pre-specified basis function, $v_{i}(u(t)$, $F)(i=1,2, \ldots, n)$ is the $i$ th weight corresponding to the RBF neural network used for PDF model, and $\omega_{0}(y, u(t), F)$ stands for either the model uncertainty or the error on the approximation of the output PDF, which is supposed to satisfy $\left|\omega_{0}(y, u(t), F)\right| \leq \delta_{0}$, where $\delta_{0}$ is assumed to be a known positive constant.

As shown in [13], the RBF basis functions are chosen as of Gaussian shapes and expressed as

$$
b_{i}(y)=\exp \left[-\left(\psi_{j}-\chi_{i}\right)^{2} / 2 \tau_{i}^{2}\right]
$$

where $\psi_{j}$ is the system input, $\chi_{i}$ and $\tau_{i}$ are respectively the centers and widths of the RBFs. 
Furthermore, we denote

$$
\begin{aligned}
B_{0}(y) & =\left[\begin{array}{llll}
b_{1}(y) & b_{2}(y) & \ldots & b_{n-1}(y)
\end{array}\right] \\
V(t)=V(u(t), F) & =\left[\begin{array}{llll}
v_{1}(u(t), F) & v_{2}(u(t), F) & \ldots & v_{n-1}(u(t), F)
\end{array}\right]^{T}
\end{aligned}
$$

and

$$
\begin{array}{lll}
\Lambda_{1}=\int_{a}^{b} B^{T}(y) B(y) d y, & \Lambda_{2}=\int_{a}^{b} B^{T}(y) b_{n}(y) d y, \\
\Lambda_{3}=\int_{a}^{b} b_{n}^{2}(y) d y \neq 0, & \Lambda_{0}=\Lambda_{1} \Lambda_{3}-\Lambda_{2}^{T} \Lambda_{2}
\end{array}
$$

In this paper, similarly to [7], we adopt the following model:

$$
\sqrt{\gamma(y, u(t), F)}=B(y) V(t)+h(V(t)) b_{n}(y)+\omega(y, u(t), F)
$$

where

$$
\begin{aligned}
B(y) & =B_{0}(y)-\frac{\Lambda_{2}}{\Lambda_{3}} b_{n}(y) \\
h(V(t)) & =\frac{1}{\Lambda_{3}}\left[-\Lambda_{2} V(t)+\sqrt{\Lambda_{2}(t)-V^{T}(t) \Lambda_{0} V(t)}\right]
\end{aligned}
$$

From the boundedness of $\omega_{0}(y, u(t), F)$ and [9], it can be assumed that $|\omega(y, u(t), F)| \leq \delta$ holds for all $\{z, u(t), F\}$, where $\delta$ is a known positive constant.

Once the square root expansion of the output PDFs has been made for the nonGaussian stochastic distribution system, the next step is to find the dynamic relationship between the control input and weights related to the PDF corresponding to a further modeling. As shown in [6], in this paper the nonlinear dynamic model will be considered as follows:

$$
\dot{V}(t)=A_{0} V(t)+G_{0} g_{0}(V(t))+B_{0} u(t)+D_{0} F+H_{0} w(t)
$$

where $V(t) \in R^{n-1}$ stands for the independent weight vectors. $A_{0}, B_{0}, G_{0}, D_{0}$, and $H_{0}$ represent the known coefficient matrices with compatible dimensions of the weight system: these matrices can be obtained either by physical modeling or the scaling estimation technique; $g_{0}(x(t))$ is a nonlinear vector function that represents the nonlinear dynamics of the weight model; $w(t)$ is the exogenous disturbance or the model perturbation.

We can see that model (6) stands for a nonlinear dynamic weight system with non-zero initial conditions. Under model (6), Eq. (4) can be rewritten as a nonlinear function of $V(t)$ as follows:

$$
\sqrt{\gamma(y, u(t), F)}=B(y) V(t)+h(V(t)) b_{n}(y)+\omega(y, u(t), F)
$$

Remark 1 Compared with the models considered in [3, 10, 19], there are the following several features: first of all, a radial basis function (RBF) neural network technique is proposed so that the PDF model is more practically reasonable; secondly, in the model adopted in [1], $\omega(y, u(t), F)$ is omitted, which can lead to the conservative result.

In the rest of this paper, the following assumptions are necessary: 
Assumption 1 For any $x_{1}(t)$ and $x_{2}(t), g(x(t))$ satisfies the following Lipschitz condition:

$$
\left\|g\left(x_{1}(t)\right)-g\left(x_{2}(t)\right)\right\| \leq\left\|U_{1}\left(x_{1}(t)-x_{2}(t)\right)\right\|
$$

where $U_{1}$ is a known constant matrix, $\|\cdot\|$ is denoted as the Euclidean norm.

Assumption 2 For any $x_{1}(t)$ and $x_{2}(t), h(V(t))$ from Eq. (5) satisfies the following Lipschitz condition:

$$
\left\|h\left(x_{1}(t)\right)-h\left(x_{2}(t)\right)\right\| \leq\left\|U_{2}\left(x_{1}(t)-x_{2}(t)\right)\right\|
$$

where $U_{2}$ is a known constant matrix.

Assumption 3 Since $V_{g}(t)$ is a known vector, we denote $y=\left\|V_{g}(t)\right\|^{2}$.

Assumption $4 F$ is an actuator fault to be compensated and rejected, so we denote

$$
F(t)= \begin{cases}F, & t \geq T \\ 0, & t<T\end{cases}
$$

where $T>0$ is an appropriate time parameter.

\section{Fault Tolerant Controller Design}

Since the measured information is the output probability distribution, after the fault has been detected and diagnosed, the following step is to design controller for nonGaussian stochastic distribution system such that the actual output PDF is made as close as possible to a given (desired) PDF. The desired PDF can be given by

$$
\sqrt{g(y)}=B(y) V_{g}(t)+h\left(V_{g}(t)\right) b_{n}(y)
$$

where $g(y)$ represents pre-specified continuous PDF, $V_{g}(t)$ stands for the desired weight vector with respect to the same basis function $B(y)$. The error between the actual output PDF and the desired PDF is defined as

$$
\begin{aligned}
\Delta e(y, t) & =\sqrt{g(y)}-\sqrt{\gamma(y, u(t), F)} \\
e(t) & =V(t)-V_{g}(t)
\end{aligned}
$$

Since $h(V(t))$ is a continuous function based on the functions continuity theory, it is noted that $\Delta_{e}(y, t) \rightarrow 0$ holds if and only if $e(t) \rightarrow 0[21,28,29]$. Therefore, the PDF tracking control in FTC context can be transformed into choosing the control input such that tracking with respect to weight vectors is realized in the presence of the fault.

For the PDF tracking control problem, meanwhile, in order to enhance robustness, the classical PID controller structure is proposed to fulfill the PDF tracking problem control. But, the classical PID controller structure cannot be applied directly since 
control input is a twin variable function of $t$ and $y$ and the non-Gaussian stochastic distribution systems can only accept one input value. As a result, we adopt the generalized PID controller as follows:

$$
u(t)=K_{P} V(t)+K_{I} \int_{0}^{t} e(y, s) d s+K_{D} \dot{V}(t)
$$

where $u(t)$ stands for the output of PID controller, $K_{P}$ is the proportional gain, $K_{I}$, $K_{D}$ are respectively the integral and derivative gains.

Based on the system (6), the nonlinear dynamic model can be transformed into the following equivalent form:

$$
E \dot{x}(t)=A x(t)+B u(t)+G g(x(t))+D F+H w(t)-N V_{g}
$$

where $x(t)=\left[\dot{V}^{T}(t), V^{T}(t), \int_{0}^{t} e^{T}(s) d s\right]^{T}$ is a new state variable, $g(z(t))=$ $\left[g^{T}(V(t)) \quad 0 \quad 0\right]^{T}$ and

$$
\begin{aligned}
& E=\left[\begin{array}{lll}
0 & 0 & 0 \\
0 & I & 0 \\
0 & 0 & I
\end{array}\right], \quad A=\left[\begin{array}{ccc}
-I & A_{0} & 0 \\
I & 0 & 0 \\
0 & I & 0
\end{array}\right], \quad B=\left[\begin{array}{c}
B_{0} \\
0 \\
0
\end{array}\right], \\
& G=\left[\begin{array}{c}
G_{0} \\
0 \\
0
\end{array}\right], \quad D=\left[\begin{array}{c}
D_{0} \\
0 \\
0
\end{array}\right], \quad H=\left[\begin{array}{c}
H_{0} \\
0 \\
0
\end{array}\right], \quad N=\left[\begin{array}{l}
0 \\
0 \\
1
\end{array}\right]
\end{aligned}
$$

Since the PID controller can be formulated as

$$
u(t)=K x(t), \quad K=\left[\begin{array}{lll}
K_{P} & K_{I} & K_{D}
\end{array}\right]
$$

with such an augment descriptor system (11), the tracking problem in FTC context can be further reduced to the stabilization control problem.

Based upon the changes of output PDFs, once the fault have been detected and diagnosed, the following step is that controller should be designed to compensate the performance losses that are caused by the fault in the system. Therefore, when the fault occurs, the fault-tolerant filter for $F$ is considered as follows:

$$
\left\{\begin{array}{l}
\dot{\hat{V}}(t)=A_{0} \hat{V}(t)+B_{0} u(t)+G_{0} g(\hat{V}(t))+H_{0} w(t)+L \varepsilon(k)+D_{0} \hat{F}(t) \\
\varepsilon(t)=\int_{a}^{b} \sigma(y)[\sqrt{\gamma(y, u(t), F)}-\sqrt{\hat{\gamma}(y, u(t))}] d y \\
\sqrt{\hat{\gamma}(y, u(t))}=B(y) \hat{V}(t)+h(\hat{V}(t)) b_{n}(y) \\
\dot{\hat{F}}=-K_{1} \hat{F}+K_{2} \varepsilon(t)
\end{array}\right.
$$

where $\hat{F}$ is the estimation of $F(t), K_{i}\left(i=1,2, K_{i}>0\right)$ are two learning operators to be determined by the proposed control method, the residual $\varepsilon(t)$ is formulated as an integral of the difference between the measured PDFs and the estimated ones, $\sigma(y)$ represents a pre-specified weighting vector defined on $[a, b]$. In fact, as the measure of the difference of two PDFs, $\varepsilon(t)$ is described as a nonlinear function of the state. Then, the residual signal can be rewritten as

$$
\varepsilon(t)=\Gamma_{1} e(t)+\Gamma_{2}[h(V(t))-h(\hat{V}(t))]+\rho(t)
$$


where

$$
\begin{aligned}
& \Gamma_{1}=\int_{a}^{b} \sigma(t) B(y) d y, \quad \Gamma_{2}=\int_{a}^{b} \sigma(t) b_{n}(y) d y, \quad e(t)=V(t)-\hat{V}(t) \\
& \|\rho(t)\|=\left\|\int_{a}^{b} \sigma(y) \omega(y, u(t), F) d y\right\| \leq \bar{\delta}=\delta \int_{a}^{b} \sigma(y) d y
\end{aligned}
$$

Defining $\tilde{F}(t)=F-\hat{F}(t)$, then it can be seen that

$$
\begin{aligned}
\dot{\tilde{F}} & =-K_{1} \tilde{F}+K_{1} F+K_{2}\left[\Gamma_{1} e(t)+\Gamma_{2} \tilde{h}+\rho(t)\right] \\
\frac{d}{d t}\left(\tilde{F}^{T} \tilde{F}\right) & =-2 \tilde{F}^{T} K_{1} \tilde{F}+2 \tilde{F}^{T} K_{1} F-2 \tilde{F}^{T}\left[K_{2} \Gamma_{1} e(t)+K_{2} \Gamma_{2} \tilde{h}+K_{2} \rho(t)\right]
\end{aligned}
$$

Substituting (12) into (11) leads to the following closed-loop system:

$$
E \dot{x}(t)=(A+B K) x(t)+G g(x(t))+D F+H w(t)-N V_{g}
$$

In [20], the feasible FTC filter design algorithm has been given. However, only the boundedness of the estimation errors could be guaranteed, which may lead to the conservative criteria for the FTC problems. Meanwhile the exogenous disturbance (or the model perturbation) is omitted. To improve the performance for the FTC, the robust $H^{\infty}$ optimization technique is introduced for the FTC based on non-Gaussian stochastic distribution system. For this purpose we construct a reference vector as follows:

$$
z(t)=C_{1} \varepsilon+C_{2} \tilde{F}=C_{1}\left(\Gamma_{1} e(t)+\Gamma_{2} \tilde{h}+\rho(t)\right)+C_{2} \tilde{F}
$$

where $C_{1}$ and $C_{2}$ stand for two selected weight matrices. By selecting $C_{1}$ and $C_{2}$ appropriately, it can be verified that $\varepsilon(t)$ and $\tilde{F}(t)$ can be bounded in a pre-specified range.

Theorem 1 For the parameters $\lambda_{i}>0(i=1,2,3)$, and $U_{1}, U_{2}$, if there exist matrices $P=P^{T}>0, R, Q$ and $\gamma_{i}>0(i=1,2,3)$ satisfying

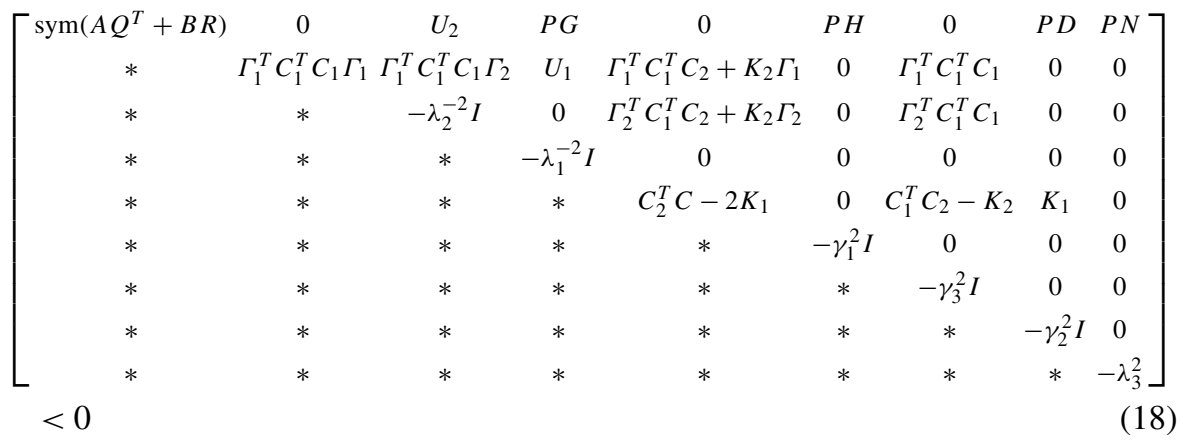

then the closed-loop system (16) is stable and satisfies

$$
\|z\|^{2} \leq \gamma_{1}^{2}\|w(t)\|^{2}+\gamma_{2}^{2}\|F\|^{2}+\gamma_{3}^{2}\|\rho(t)\|^{2}
$$

The PID control gain $K$ can be solved via $R=K Q^{T}$. 
Proof Define the Lyapunov candidate function as follows:

$$
\begin{aligned}
V(x(t), t)= & x^{T}(t) P \operatorname{Ex}(t)+\tilde{F}^{T} \tilde{F}+\frac{1}{\lambda_{1}^{2}} \int_{0}^{t}\left[\left\|U_{1} x(\tau)\right\|^{2}-\|g(x(\tau))\|^{2}\right] d \tau \\
& +\frac{1}{\lambda_{2}^{2}} \int_{0}^{t}\left[\left\|U_{2} x(\tau)\right\|^{2}-\|\tilde{h}(x(\tau))\|^{2}\right] d \tau
\end{aligned}
$$

Along the trajectories of (16), it can be verified that

$$
\begin{aligned}
\dot{V}(x(t), t)= & 2 x^{T}(t) P E \dot{x}(t)+\dot{\tilde{F}}^{T} \tilde{F}+\frac{1}{\lambda_{2}^{2}}\left[\left\|U_{2} x(t)\right\|^{2}-\|\tilde{h}\|^{2}\right] \\
& +\frac{1}{\lambda_{1}^{2}}\left[\left\|U_{1} x(\tau)\right\|^{2}-\|g(x(\tau))\|^{2}\right] \\
= & 2 x^{T}(t) P\left[(A+B K) x(t)+G g(x(t))+D F+H w(t)+N V_{g}\right] \\
& -2 \tilde{F}^{T}\left[K_{2} \Gamma_{1} e(t)+K_{2} \Gamma_{2} \tilde{h}+K_{2} \rho(t)\right]-2 \tilde{F}^{T} K_{1} \tilde{F}+2 \tilde{F}^{T} K_{1} F \\
& +\frac{1}{\lambda_{2}^{2}}\left[\left\|U_{2} x(t)\right\|^{2}-\|\tilde{h}\|^{2}\right]+\frac{1}{\lambda_{1}^{2}}\left[\left\|U_{1} x(\tau)\right\|^{2}-\|g(x(\tau))\|^{2}\right] \\
= & x^{T}(t) \operatorname{sym}(P(A+B K)) x(t)+2 x^{T}(t) P G g(x(t))+2 x^{T}(t) P D F \\
& +2 x^{T}(t) P H w(t)+2 x^{T}(t) P N V_{g}-2 \tilde{F} K_{2} \Gamma_{1} e(t)-2 \tilde{F}^{T} K_{2} \Gamma_{2} \tilde{h} \\
& -2 \tilde{F}^{T} K_{2} \rho(t)-2 \tilde{F}^{T} K_{1} \tilde{F}+2 \tilde{F}^{T} K_{1} F+\frac{1}{\lambda_{2}^{2}}\left[\left\|U_{2} x(t)\right\|^{2}-\|\tilde{h}\|^{2}\right] \\
& +\frac{1}{\lambda_{1}^{2}}\left[\left\|U_{1} x(\tau)\right\|^{2}-\|g(x(\tau))\|^{2}\right]
\end{aligned}
$$

For $\|z(t)\|^{2}$, we have

$$
\|z(t)\|^{2}=\left[C_{1}\left(\Gamma_{1} e(t)+\Gamma_{2} \tilde{h}+\rho(t)\right)+C_{2} \tilde{F}\right]^{T}\left[C_{1}\left(\Gamma_{1} e(t)+\Gamma_{2} \tilde{h}+\rho(t)\right)+C_{2} \tilde{F}\right]
$$

Furthermore, we denote the following auxiliary function as the performance index:

$$
J=\int_{0}^{\infty}\left[\|z\|^{2}-\gamma_{1}^{2}\|w(t)\|^{2}-\gamma_{2}^{2}\|F\|^{2}-\gamma_{3}^{2}\|\rho(t)\|^{2}\right] d t
$$

Then we have the following result:

$$
\begin{aligned}
J & =\int_{0}^{t}\left[\|z\|^{2}-\gamma_{1}^{2}\|w(t)\|^{2}-\gamma_{2}^{2}\|F\|^{2}-\gamma_{3}^{2}\|\rho(t)\|^{2}\right] d t \\
& =\int_{0}^{t}\left[\|z\|^{2}-\gamma_{1}^{2}\|w(t)\|^{2}-\gamma_{2}^{2}\|F\|^{2}-\gamma_{3}^{2}\|\rho(t)\|^{2}+\dot{V}(x(t), t)\right] d t-V(x(t), t) \\
& \leq \int_{0}^{t}\left[\|z\|^{2}-\gamma_{1}^{2}\|w(t)\|^{2}-\gamma_{2}^{2}\|F\|^{2}-\gamma_{3}^{2}\|\rho(t)\|^{2}+\dot{V}(x(t), t)\right] d t
\end{aligned}
$$

According to (15)-(21), it can be verified that 


$$
\begin{aligned}
\|z\|^{2}- & \gamma_{1}^{2}\|w(t)\|^{2}-\gamma_{2}^{2}\|F\|^{2}-\gamma_{3}^{2}\|\rho(t)\|^{2}+\dot{V}(x(t), t) \\
= & \left.C_{1}\left(\Gamma_{1} e(t)+\Gamma_{2} \tilde{h}+\rho(t)\right)+C_{2} \tilde{F}\right]^{T}\left[C_{1}\left(\Gamma_{1} e(t)+\Gamma_{2} \tilde{h}+\rho(t)\right)+C_{2} \tilde{F}\right] \\
& -\gamma_{1}^{2}\|w(t)\|^{2}-\gamma_{2}^{2}\|F\|^{2}-\gamma_{3}^{2}\|\rho(t)\|^{2} \\
& +x^{T}(t) \operatorname{sym}(P(A+B K)) x(t)+2 x^{T}(t) P G g(x(t))+2 x^{T}(t) P D F \\
& +2 x^{T}(t) P H w(t)-2 x^{T}(t) P N V_{g}-2 \tilde{F} K_{2} \Gamma_{1} e(t)-2 \tilde{F}^{T} K_{2} \Gamma_{2} \tilde{h} \\
& -2 \tilde{F}^{T} K_{2} \rho(t)-2 \tilde{F}^{T} K_{1} \tilde{F}+2 \tilde{F}^{T} K_{1} F \\
& +\frac{1}{\lambda_{1}^{2}}\left[\left\|U_{1} x(\tau)\right\|^{2}-\|g(x(\tau))\|^{2}\right]+\frac{1}{\lambda_{2}^{2}}\left[\left\|U_{2} x(t)\right\|^{2}-\|h(x(t))\|^{2}\right] \\
\leq & \left.C_{1}\left(\Gamma_{1} e(t)+\Gamma_{2} \tilde{h}^{2}+\rho(t)\right)+C_{2} \tilde{F}\right]^{T}\left[C_{1}\left(\Gamma_{1} e(t)+\Gamma_{2} \tilde{h}+\rho(t)\right)+C_{2} \tilde{F}\right] \\
& -\gamma_{1}^{2}\|w(t)\|^{2}-\gamma_{2}^{2}\|F\|^{2}-\gamma_{3}^{2}\|\rho(t)\|^{2}+x^{T}(t) \operatorname{sym}(P(A+B K)) x(t) \\
& +2 x^{T}(t) P G g(x(t))+2 x^{T}(t) P D F+2 x^{T}(t) P H w(t) \\
& +\lambda_{3}^{-2} x^{T}(t) P N N^{T} P^{T} x(t)-\lambda_{3}^{2} y-2 \tilde{F} K_{2} \Gamma_{1} e(t)-2 \tilde{F}^{T} K_{2} \Gamma_{2} \tilde{h} \\
& -2 \tilde{F}^{T} K_{2} \rho(t)-2 \tilde{F}^{T} K_{1} \tilde{F}+2 \tilde{F}^{T} K_{1} F \\
& +\frac{1}{\lambda_{1}^{2}}\left[\left\|U_{1} x(\tau)\right\|^{2}-\|g(x(\tau))\|^{2}\right]+\frac{1}{\lambda_{2}^{2}}\left[\left\|U_{2} x(t)\right\|^{2}-\|h(x(t))\|^{2}\right] \\
= & \xi \xi^{T} \Xi
\end{aligned}
$$

where

$$
\begin{aligned}
& \Xi=\left[\begin{array}{cccccccc}
\chi_{11} & 0 & 0 & P G & 0 & P H & 0 & P D \\
* & \Gamma_{1}^{T} C_{1}^{T} C_{1} \Gamma_{1} \Gamma_{1}^{T} C_{1}^{T} C_{1} \Gamma_{2} & 0 & \Gamma_{1}^{T} C_{1}^{T} C_{2}+K_{2} \Gamma_{1} & 0 & \Gamma_{1}^{T} C_{1}^{T} C_{1} & 0 \\
* & * & -\lambda_{2}^{-2} I & 0 & \Gamma_{2}^{T} C_{1}^{T} C_{2}+K_{2} \Gamma_{2} & 0 & \Gamma_{2}^{T} C_{1}^{T} C_{1} & 0 \\
* & * & * & -\lambda_{1}^{-2} I & 0 & 0 & 0 & 0 \\
* & * & * & * & C_{2}^{T} C-2 K_{1} & 0 & C_{1}^{T} C_{2}-K_{2} & K_{1} \\
* & * & * & * & * & -\gamma_{1}^{2} I & 0 & 0 \\
* & * & * & * & * & * & -\gamma_{3}^{2} I & 0 \\
* & * & * & * & * & * & * & -\gamma_{2}^{2} I
\end{array}\right] \\
& \chi_{11}=\operatorname{sym}(P(A+B K))+\lambda_{3}^{-2} P N N^{T} P^{T}+\lambda_{1}^{-2} U_{1}^{T} U_{1}+\lambda_{2}^{-2} U_{2}^{T} U_{2} \\
& \xi=\left[\begin{array}{llllllll}
x^{T}(t) & e^{T}(t) & \tilde{h}^{T} & g & \tilde{F}^{T} & w^{T} & \rho & F^{T}
\end{array}\right]^{T}
\end{aligned}
$$

Denoting $Q=P^{-1}$, by pre-multiplying $\operatorname{diag}\left(P^{-1} I I I I I I I I I\right)$ and postmultiplying $\operatorname{diag}\left(P^{-1} \text { I I I I I I I }\right)^{T}$ to (23) and using the Schur complement formula, (18) can be obtained.

Thus, $J<0$ holds because of (18). From the definition (21) of $J$ and (18), it can be shown that

$$
\|z\|^{2} \leq \gamma_{1}^{2}\|w(t)\|^{2}+\gamma_{2}^{2}\|F\|^{2}+\gamma_{3}^{2}\|\rho(t)\|^{2}
$$

On the other hand, in the absence of the exogenous inputs, $\Xi<0$ implies that $\dot{V}(x(t), t)<0$, which means that the closed-loop system is stable. 
In Theorem 1, an available FTC result has been obtained based on filtering methodology. The proposed result proved an LMI-based solution to design the controller with which not only the fault can be compensated but also the disturbance can be attenuated.

\section{Simulation}

To further illustrate the above-mentioned approach, an application to paper-making process. It is supposed that the output PDF can be approximated using three-layer neural networks with three radial basis activation functions with the following initial condition:

$$
y \in[0,1], \quad \chi_{1}=0.2, \quad \chi_{2}=0.5, \quad \chi_{3}=0.8, \quad \tau_{1}=\tau_{2}=\tau_{3}=0.05
$$

It is assumed that the weight system is formulated by (6) with the following coefficient matrices:

$$
\begin{array}{ll}
A_{0}=\left[\begin{array}{cc}
-0.5 & 0 \\
0 & -1
\end{array}\right], \quad B_{0}=\left[\begin{array}{cc}
-0.2 & 0 \\
0 & -0.1
\end{array}\right], \quad G_{0}=\left[\begin{array}{ll}
1 & 0 \\
0 & 1
\end{array}\right], \\
H_{0}=\left[\begin{array}{cc}
1 & 0 \\
0 & -1
\end{array}\right], & D_{0}=\left[\begin{array}{l}
0.5 \\
0.5
\end{array}\right]
\end{array}
$$

Let the model error exist and satisfy $\|\omega(y, u(t), F)\| \leq 0.001$.

The parameters can be chosen as $\lambda_{1}=\lambda_{2}=\lambda_{3}=1$. The fault is supposed to be as the following:

$$
F(t)= \begin{cases}0.5, & t \geq 10 \\ 0, & t<10\end{cases}
$$

When the generalized PID controller is applied, the response to the residual signal by using the diagnostic filter is as shown in Fig. 1. It is demonstrated that the response of the residual signal converges to zero asymptotically. Figure 2 demonstrates the tracking error of the fault, which shows that the fault can be well estimated through the fault diagnostic filter. Furthermore, we consider the time-varying fault described as

$$
F(t)= \begin{cases}1+0.02 \sin (0.2 t), & t \geq 10 \\ 0, & t<10\end{cases}
$$

Fig. 1 Response of the residual

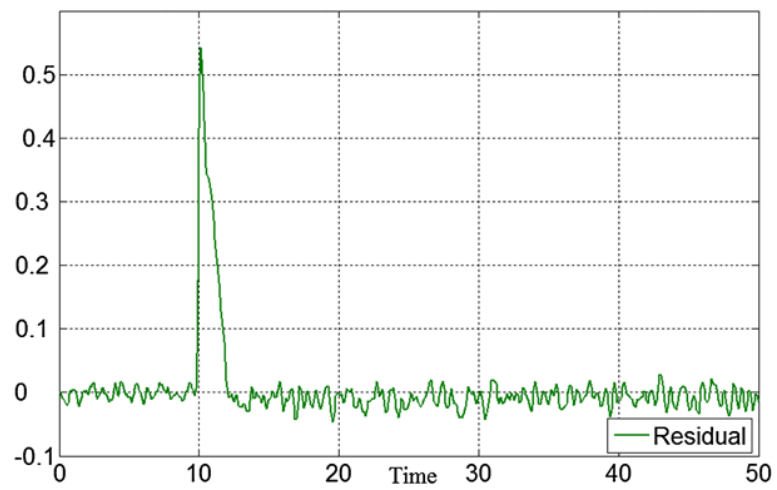


Fig. 2 Response of diagnosis observer and step fault

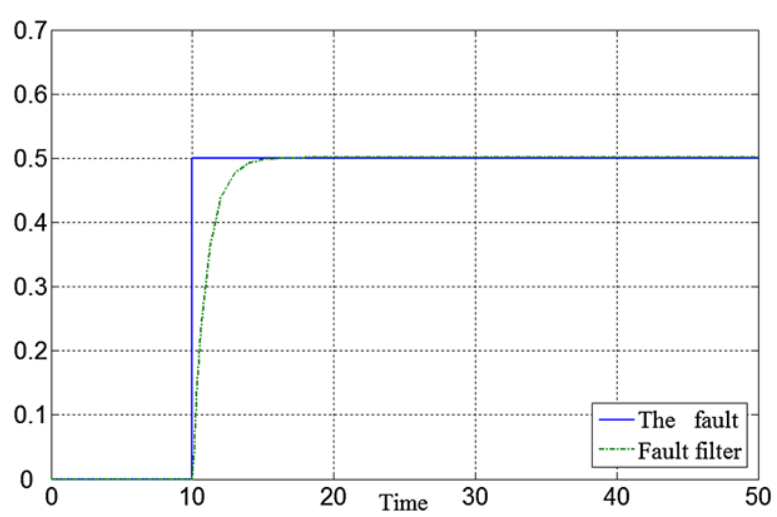

Fig. 3 Response of diagnosis observer and time-varying fault

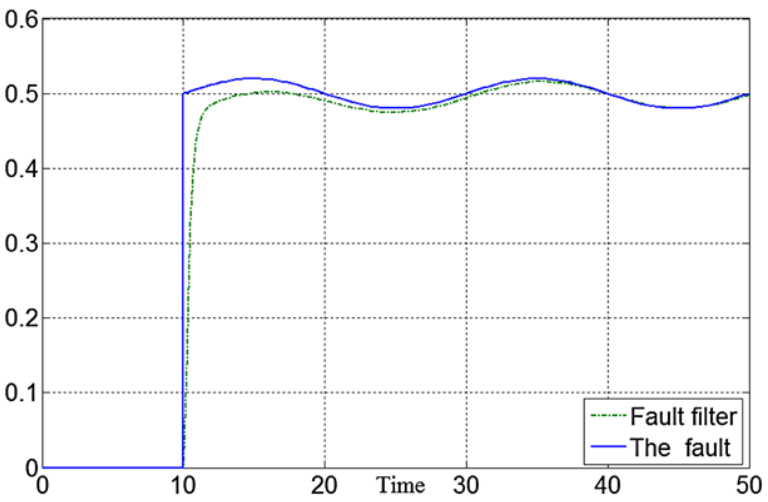

Figure 3 shows the response of the residual signal by using diagnostic filter. Simulation demonstrates that the fault can be well compensated, and at the same time satisfactory tracking performance, stability and robustness are achieved.

\section{Conclusion}

In this paper, a new FTC algorithm is investigated for non-Gaussian stochastic distribution system using (RBF) neural network. Differently from the conventional FTC methods, the measured information is the output PDF rather than its instant values, where the RBF neural network technique is introduced so that the output PDFs can be formulated by the dynamic weights. Then, based on $H^{\infty}$ optimization techniques and PID controller, the concerned FTC problem can be transformed into a classical nonlinear FTC problem subject to nonlinear systems with both modeling error and the fault. In terms of LMI techniques, a new control method is given so that the fault can be compensated or rejected. The simulation of the illustrated example demonstrates the efficiency of the proposed approach.

Open Access This article is distributed under the terms of the Creative Commons Attribution License which permits any use, distribution, and reproduction in any medium, provided the original author(s) and the source are credited. 


\section{References}

1. M. Blanke, M. Staroswiecki, Diagnosis and Fault-Tolerant Control, 2nd edn. (Springer, Berlin, 2006)

2. L.G. Crespo, J.Q. Sun, Non-linear stochastic control via stationary response design. Probab. Eng. Mech. 18, 73-86 (2003)

3. L. Guo, H. Wang, Fault detection and diagnosis for general stochastic systems using B-spline expansions and nonlinear filters. IEEE Trans. Circuits Syst. I, Fundam. Theory Appl. 52(8), 1644-1652 (2005)

4. L. Guo, H. Wang, Observer-based optimal fault detection and diagnosis using conditional probability distribution. IEEE Trans. Signal Process. 54(10), 3712-3719 (2006)

5. Y. Guo, B. Yang, P. Shi, Delay-dependent adaptive reconfiguration control in the presence of input saturation and actuator faults. Int. J. Innov. Comput. Inf. Control 6(4), 1873-1882 (2010)

6. Z.H. Hu, Z.Z. Han, Z.H. Tian, Fault detection and diagnosis for singular stochastic systems via B-spline expansions. ISA Trans. 48, 519-524 (2009)

7. Z.H. Hu, Z.Z. Han, Z.H. Tian, Fault diagnosis for singular stochastic system. J. Shanghai Jiaotong Univ. 16(4), 497-501 (2011)

8. R. Isermann, Fault-Diagnosis Systems: An Introduction from Fault Detection to Fault Tolerance (Springer, Berlin, 2006)

9. T. Li, Y.C. Zhang, Fault detection and diagnosis for stochastic systems via output PDFs. J. Franklin Inst. 348, 1140-1152 (2011)

10. T. Li, Y. Yi, L. Guo, H. Wang, Delay-dependent fault detection and diagnosis using B-spline neural networks and nonlinear filters for time-delay stochastic systems. Neural Comput. Appl. 17, 405-411 (2008)

11. H. Li, Y.P. Zhang, H.Q. Zheng, Gear fault detection and diagnosis under speed-up condition based on order cepstrum and radial basis function neural network. J. Mech. Sci. Technol. 23, 2780-2789 (2009)

12. M. Mahmoud, J. Jiang, Y. Zhang, Stochastic stability analysis of fault-tolerant control systems in the presence of noise. IEEE Trans. Autom. Control 46(11), 1810-1815 (2001)

13. L. Meng, B. Jiang, Robust active fault-tolerant control for a class of uncertain nonlinear systems with actuator faults. Int. J. Innov. Comput. Inf. Control 6(6), 2637-2644 (2010)

14. S.K. Nguang, P. Shi, S. Ding, Fault detection for uncertain fuzzy systems: an LMI approach. IEEE Trans. Fuzzy Syst. 15(6), 1251-1262 (2007)

15. A. Okatan, C. Hajiyev, Fault detection in sensor information fusion Kalman filter. AEÜ, Int. J. Electron. Commun. 63, 762-768 (2009)

16. K. Salahshoor, M. Kordestani, M.S. Khoshro, Fault detection and diagnosis of an industrial steam turbine using fusion of SVM (support vector machine) and ANFIS (adaptive neuro-fuzzy inference system) classifiers. Energy 35, 5472-5482 (2010)

17. B. Shen, H.S. Shu, $H^{\infty}$ filtering for nonlinear discrete-time stochastic systems with randomly varying sensor delays. Automatica 45, 1032-1037 (2009)

18. Z. Simeu-Abazi, M. Di Mascolo, M. Knotek, Fault diagnosis for discrete event systems: modelling and verification. Reliab. Eng. Syst. Saf. 95, 369-378 (2010)

19. Z. Skaf, H. Wang, L. Guo, Fault tolerant control based on stochastic distribution via RBF neural network. J. Syst. Eng. Electron. 22(1), 63-69 (2011)

20. X.B. Sun, H. Yue, H. Wang, Modelling and control of the flame temperature distribution using probability density function shaping. Trans. Inst. Meas. Control 28(5), 401-428 (2006)

21. C. Turchetti, P. Crippa, M. Pirani, G. Biagetti, Representation of nonlinear random transformations by non-Gaussian stochastic neural networks. IEEE Trans. Neural Netw. 19(6), 1033-1060 (2008)

22. H. Wang, Applying observer based FDI techniques to detect faults in dynamic and bounded stochastic distributions. Int. J. Control 73(15), 1424-1436 (2000)

23. H. Wang, Bounded Dynamic Stochastic Distributions Modeling and Control (Springer, Berlin, 2000)

24. H. Wang, An online estimation algorithm for the unknown probability density functions of random parameters in stochastic ARMAX systems. IEEE Trans. Autom. Control 153, 462-468 (2006)

25. H. Wang, P. Afschar, H. Yue, ILC-based generalised PI control for output PDF of stochastic system using LMI and RBF neural networks, in Proc. of the IEEE Conference on Decision and Control (2006), pp. 5048-5053

26. Y. Wang, W. Wang, D. Wang, LMI approach to design fault detection filter for discrete-time switched systems with state delays. Int. J. Innov. Comput. Inf. Control 6(1), 387-398 (2010)

27. Y. Xu, B. Jiang, G. Tao, Z. Gao, Fault accommodation for near space hypersonic vehicle with actuator fault. Int. J. Innov. Comput. Inf. Control 7(5), 2187-2200 (2010) 
28. R.N. Yang, P. Shi, Exponential stability on stochastic neural networks with discrete interval and distributed delays. IEEE Trans. Neural Netw. 21(1), 169-175 (2010)

29. R.N. Yang, H.J. Gao, P. Shi, Novel robust stability criteria for stochastic Hopfield neural networks with time delays. IEEE Trans. Syst. Man Cybern., Part B, Cybern. 39(11), 467-474 (2009)

30. R.N. Yang, P. Shi, G.P. Liu, Filtering for discrete-time networked nonlinear systems with mixed random delays and packet dropouts. IEEE Trans. Autom. Control 56(11), 2655-2660 (2011)

31. L.N. Yao, H. Wang, Robust fault diagnosis for non-Gaussian stochastic systems based on the rational square-root approximation model. Sci. China Ser. F 51(9), 1281-1290 (2008)

32. Y. Yi, H. Shen, L. Guo, Statistic PID tracking control for non-Gaussian stochastic systems based on T-S fuzzy model. Int. J. Autom. Comput. 6(1), 81-87 (2009)

33. Y. Yi, T.P. Zhang, L. Guo, Multi-objective PID control for non-Gaussian stochastic distribution system based on two-step intelligent models. Sci. China Ser. Inf. Sci. 52(10), 1754-1765 (2009)

34. Y. Zhang, Z. Wang, J. Zhang, J. Ma, Fault detection based on discriminant analysis theory in power systems. ICIC Express Lett. 4(3), 809-814 (2010)

35. J.L. Zhou, D.H. Zhou, Distribution function tracking filter design using hybrid characteristic functions. Automatica 46, 101-109 (2010) 